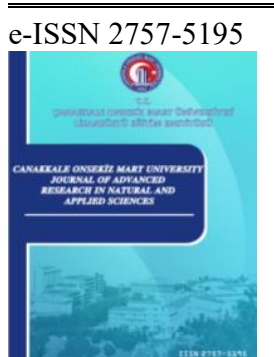

Çanakkale Onsekiz Mart University

Journal of Advanced Research in Natural and Applied Sciences

Open Access

\title{
O-C Analysis of Algol-type Eclipsing Binary UU And
}

\author{
Oğuz Öztürk ${ }^{1,2, *}$ \\ ${ }^{1}$ Astrophysics Research Centre and Observatory, Çanakkale Onsekiz Mart University, Çanakkale, Turkey \\ ${ }^{2}$ Department of Physics, Faculty of Arts and Sciences, Çanakkale Onsekiz Mart University, Çanakkale, Turkey
}

\begin{abstract}
Article History
Received: $\quad 19.03 .2020$

Accepted: $\quad 15.09 .2020$

Published: $\quad 29.12 .2020$
\end{abstract}

Research Article

\begin{abstract}
Using all available minimum times of Algol-type eclipsing binary UU And, Observed(O) - Calculated (C) based orbital period analysis of UU And is presented in this study. O-C diagram of UU And is represented by cyclic variation superimposed on an upward parabolic variation. The upward parabolic variation was modelled by assuming non-conservative mass transfer from the less massive to the more massive component to explain the secular period increase of the system. The rate of fractional mass transfer $(\beta)$ was calculated to be 0.947 . Cyclic O-C variation at period of 14 years was detected in the diagram. Light travel time effect (LTTE) caused by additional body in the system was assumed in order to explain the cyclic $\mathrm{O}-\mathrm{C}$ variation. Using the mass function obtained from $\mathrm{O}-\mathrm{C}$ analysis, the possible mass of the third component was found to be in the order of solar mass. Such a massive additional body show itself distinctly in the light curve of UU And. On the contrary, no light contribution from an additional body has been detected in the literature so far. Therefore, we conclude that precise high resolution spectroscopic observations are required in order to clarify the existence of a third body and/or magnetic cycle, which may also give rise to cyclic O-C variation in the binary star system.
\end{abstract}

Keywords - Techniques: photometric, stars: individual: UU And, eclipsing binary star systems.

\section{Introduction}

According to the catalogue presented by Brancewicz \& Dworak (1980), UU And (TYC 2275-911-1, 2MASS J00434508+3056197) is a semidetached Algol-type eclipsing binary star system consisting of a F5 type primary and K5IV type secondary components.

Up to the present, O-C based orbital period analysis studies have only been presented by Qian (2001) and Manzoori et al. (2015). Qian (2001) made a period analysis of UU And using 150 visual and photographic primary minimum times. Qian (2001) suggested secular period decrease. Rate of the period decrease was calculated to be $-4.76 \times 10^{-7}$ days/yr. Secular mass and angular momentum loss were suggested as possible mechanisms of the orbital period decrease. Manzoori et al. (2015) re-examined the O-C changes of UU And with 183 minimum times observed from 1911 to 2008. Out of 183, 36 were photoelectric (Pe) minimum times observed under the WASP survey (Pollacco et al., 2006). Manzoori et al. (2015) proposed secular orbital period increase at rate of $4.63 \times 10^{-7}$ days /yr. Secular mass transfer between components was attributed by Manzoori et al. (2015). Manzoori et al. (2015) also detected a cyclic O-C change over a period of $18 \mathrm{yr}$, suggesting that magnetic activity in the system was the most plausible mechanism responsible for the cyclic O-C change.

As seen above, there are two incompatible results in the literature obtained from O-C based orbital period analysis of UU And. However, 27 CCD and Pe minimum times have been published since 2008. Therefore, it is necessary to re-investigate the $\mathrm{O}-\mathrm{C}$ variation of UU And using precise new CCD and Pe times along with

\footnotetext{
1 (D) oguzozturk@comu.edu.tr

${ }^{*}$ Corresponding Author
} 
previously published minimum times. We present a more recent O-C analysis of UU And using all minimum times published in the literature so far. The minimum times used in our analysis were observed from 1911 to 2018.

\section{Materials and Methods}

Firstly, all available minimum times were collected from the literature. Our observational data covers 174 visual, 5 photographic and 78 CCD \& Pe minimum times of UU And, which were observed from 1911 to 2018 .

$\mathrm{O}-\mathrm{C}$ variation of UU And was re-examined using the weighted O-C method. During the analysis, since the precision of observed minimum times is dependent on the type of observation, 1 weights are given to visual observations, 5 weights are given to photographic observations and 10 weights were given to CCD \& Pe observations.

$\mathrm{O}-\mathrm{C}$ values were calculated using the linear light element equation below, where the orbital period $(P=$ $\left.1^{\mathrm{d}} .486324\right)$ was taken from Kreiner $(2004)$ and the conjunction time $\left(T_{0}(\mathrm{HJD})=2441650.338\right)$ is a primary minimum time in our minimum time data set.

$$
C_{1}=\text { HJD } 2441650.338+1^{\mathrm{d}} .486324 \times E
$$

Upward parabolic change is detected in the diagram of UU And (see Figure 1).To find the quadratic ephemeris of the system, the MATLAB code presented by Zasche et al. (2009) was used. Then the ephemeris was derived as follows:

$$
C_{2}=\text { HJD 2441650.3132(22) }+1^{\mathrm{d}} .4863031(3) \times E+3^{\mathrm{d}} .74(21) \times 10^{-10} \times E^{2}
$$

The positive quadratic term $Q=3^{d} .74(21) \times 10^{-10}$ days gives the rate of period increase to be $\dot{P}=0.016(6)$ $\mathrm{s} / \mathrm{yr}$. Discussion of the secular period increase is presented in the final section of this paper.

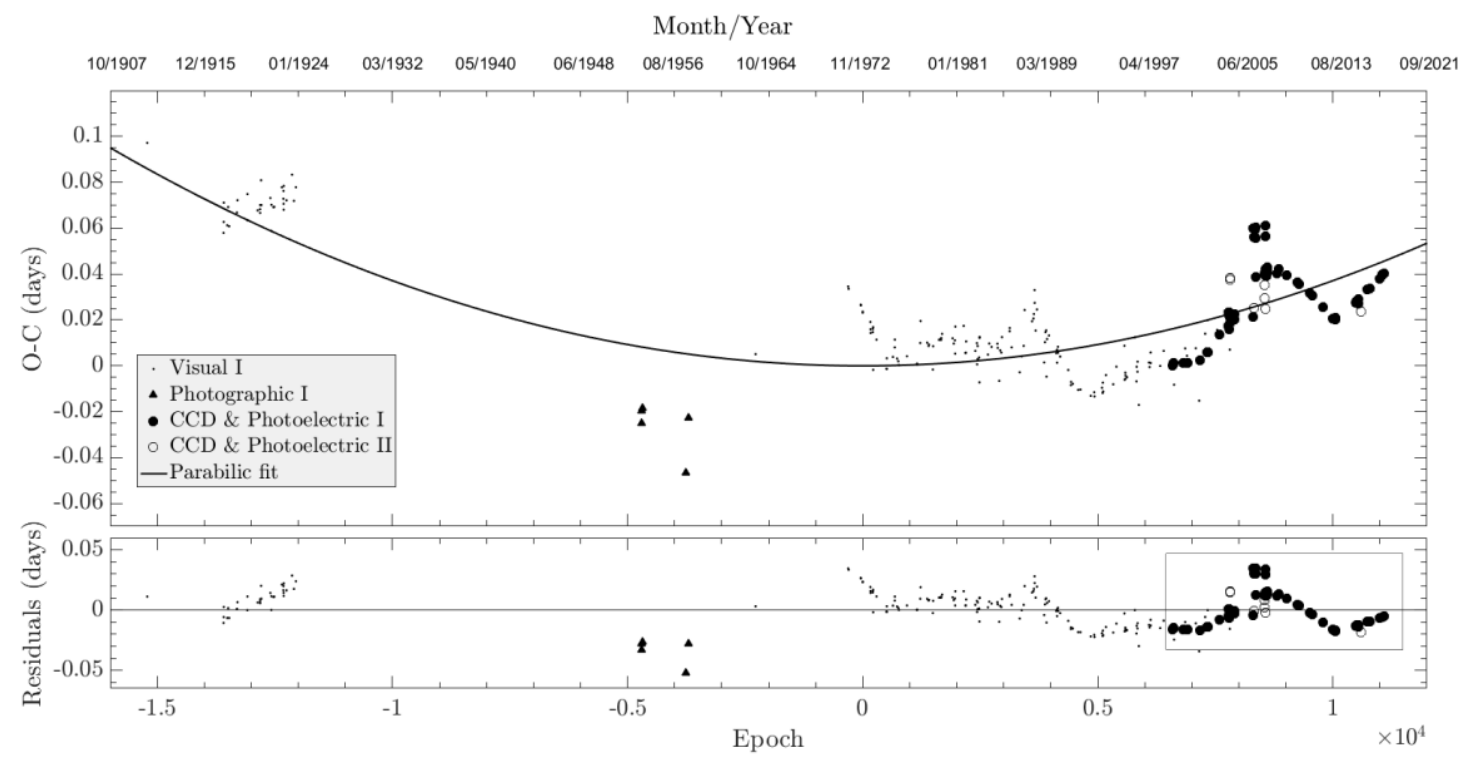

Figure 1. O-C variation of UU And. Solid line in upper panel represents parabolic fit obtained. Residuals are shown in lower panel of diagram. 
As is seen from the residuals, $\mathrm{O}-\mathrm{C}$ data also show sinusoidal period oscillation. The light-time effect (LTTE) caused by additional body may give rise to this cyclic variation. Figure 1 shows a 48-year gap from 1924 to 1972. In addition to this, most of the data before 2005 is visual and photographic. Therefore, in order to examine the sinusoidal variation accurately, we decided to use only CCD and Pe data, which can be seen in the box in the residuals. The LTTE equation (Irwin, 1959) given below was fitted using the MATLAB code presented by Zasche et al. (2009), where $\Delta t, a_{12}, c, i_{12}, e_{12}, \omega_{12}$, and $v_{12}$ are, respectively, the time delay due to LTTE, semi-major axis of the additional body, speed of light, orbital inclination of additional body, eccentricity of the additional body, longitude of periastron, and the true anomaly.

$$
\Delta t=\frac{a_{12} \sin i_{12}}{c}\left\{\frac{1-e_{12}^{2}}{1+\cos v_{12}} \sin \left(v_{12}+\omega_{12}\right)+e_{12} \cos \omega_{12}\right\}
$$

Sinusoidal variation of the CCD and Pe O-C residuals together with the best theoretical LTTE model is given in Figure 2.

Results of our O-C analysis are given in Table 1. In this table, $f(M)$, which is defined in equation (2.4), is well-known mass function of the triple system calculated from our analysis.

$$
f(M)=\frac{M_{3}^{3} \sin ^{3} i_{12}}{\left(M_{1}+M_{2}+M_{3}\right)^{2}}=\frac{a_{12}^{3} \sin ^{3} i_{12}}{P_{12}^{2}}
$$

Here, $P_{12}$ is the period of the additional body and $M_{3}$ is the mass of the additional body. Units of $P_{12}$ and $M_{3}$ are years and stellar mass, respectively. Detailed discussion on the LTTE is presented in the last section of this paper.

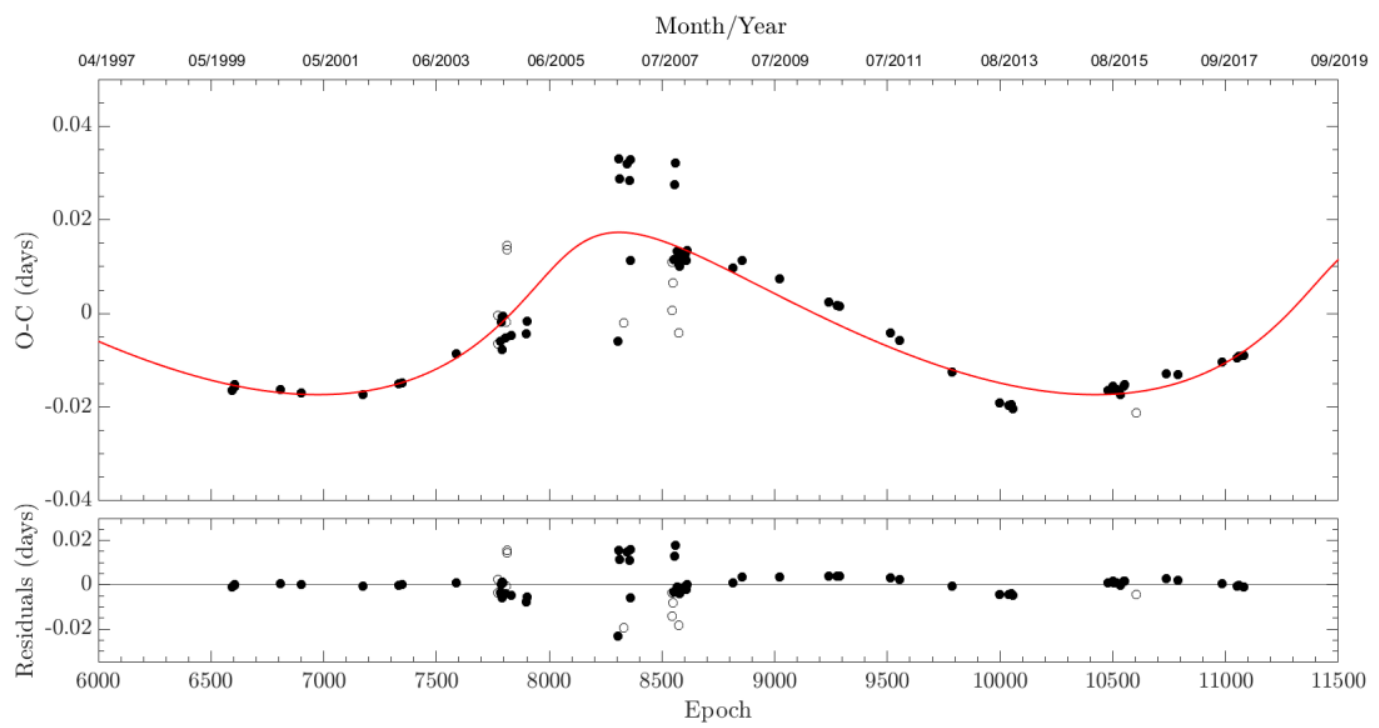

Figure 2. LTTE effect of CCD and Pe O-C data on UU And. Solid red line represents best LTTE model obtained from O-C analysis. Lower panel shows residuals from theoretical curve. 
Table 1

Parameters obtained from O-C analysis of UU And.

\begin{tabular}{ll}
\hline Parameter & \multicolumn{1}{c}{ Value } \\
\hline$T_{0}(\mathrm{HJD})$ & $2441650.3132(0.0022)$ \\
$P$ (days) & $1.4863031(0.0000003)$ \\
$Q \times 10^{-10}($ days $)$ & $3.74(0.21)$ \\
$A$ (days) & $0.017(0.002)$ \\
$a_{12} \sin i_{12}(\mathrm{AU})$ & $3.24(0.26)$ \\
$e_{12}$ & $0.49(0.12)$ \\
$\omega_{12}\left({ }^{\circ}\right)$ & $40(25)$ \\
$P_{12}($ years $)$ & $14.04(0.61)$ \\
$f(M)\left(\mathrm{M}_{\odot}\right)$ & $0.173(0.037)$ \\
$M_{3}\left(\mathrm{M}_{\odot}\right)$ for $i_{12}=90^{0}$ & $1.34(0.13)$ \\
$M_{3}\left(\mathrm{M}_{\odot}\right)$ for $i_{12}=60^{\circ}$ & $1.62(0.15)$ \\
\hline
\end{tabular}

\section{Results and Discussion}

The recent $\mathrm{O}-\mathrm{C}$ analysis of UU And is presented in this study. We found that orbital period of UU And is increasing at a $\dot{P}=0.016(6) \mathrm{s} / \mathrm{yr}$ rate (see Section 2). Manzoori et al. (2015) suggested possible magnetic activity in the system since the system consists of a F5 type primary and K5IV type secondary. Therefore, a possible mechanism, which produces upward parabolic variation, may be non-conservative (or Alfvén driven) mass transfer between components since some fraction of the total mass can be lost from UU And via magnetic winds (Tout \& Hall 1991). In this case, we followed the procedure presented by Erdem \& Öztürk (2014) to explain the observed period increase of UU And and used the equations below.

$$
\begin{aligned}
& \frac{\dot{P}}{P}=\left\{2\left\{\frac{10 R_{2}}{d}\right\} \frac{M_{1}+M_{2}}{M_{1} M_{2}}-\frac{2}{M_{1}+M_{2}}\right\} \dot{M}+3 \frac{\left(M_{1}-M_{2}\right)}{M_{1} M_{2}} \dot{M}_{2} \\
& \dot{M} \leq(1-\beta) \dot{M}_{2}=\frac{(\beta-1)}{\beta} \dot{M}_{1}
\end{aligned}
$$

where $M_{1}, M_{2}, R_{2}, d, \dot{M}<0, \dot{M}_{1}>0, \dot{M}_{2}=\dot{M}-\dot{M}_{1}<0$ and $\beta\left(0<\beta<\beta_{c r i},\left(\beta_{c r i}\right.\right.$ is the maximum possible value of the fractional mass transfer rate for systems showing period increase)) are: masses of primary and secondary components, radius of secondary component, distance between components, mass loss rate from UU And, rate of mass transfer, mass loss rate from less massive component, and the fractional mass transfer parameter, respectively. Term $10 R_{2}$ in Eq. (3.1) represents the approximate value of Alfvén radius (Tout \& Hall 1991) for Alfvén driven mass transfer in Algols (Erdem \& Öztürk 2014). $\beta_{c r i}$ was calculated from Eq. (3.1) and Eq. (3.2) to be 0.947. In this calculation, while $\dot{P}=0.016(6) \mathrm{s} / \mathrm{yr}$ and $P=1.4863031$ (3) days are taken from the results of $\mathrm{O}-\mathrm{C}$ analysis in this study, $M_{1}=1.62(1) \mathrm{M}_{\odot}, M_{2}=0.76(24) \mathrm{M}_{\odot}$ and $R_{2}=$ 2.28(54) $\mathrm{R}_{\odot}$ are taken from Manzoori et al. (2015), and $d=7.23 \mathrm{AU}$ is taken from Brancewicz \& Dworak (1980). Figure 3 shows the change in the fractional mass transfer rate $(\beta)$ with respect to $\dot{M}_{2}$. 


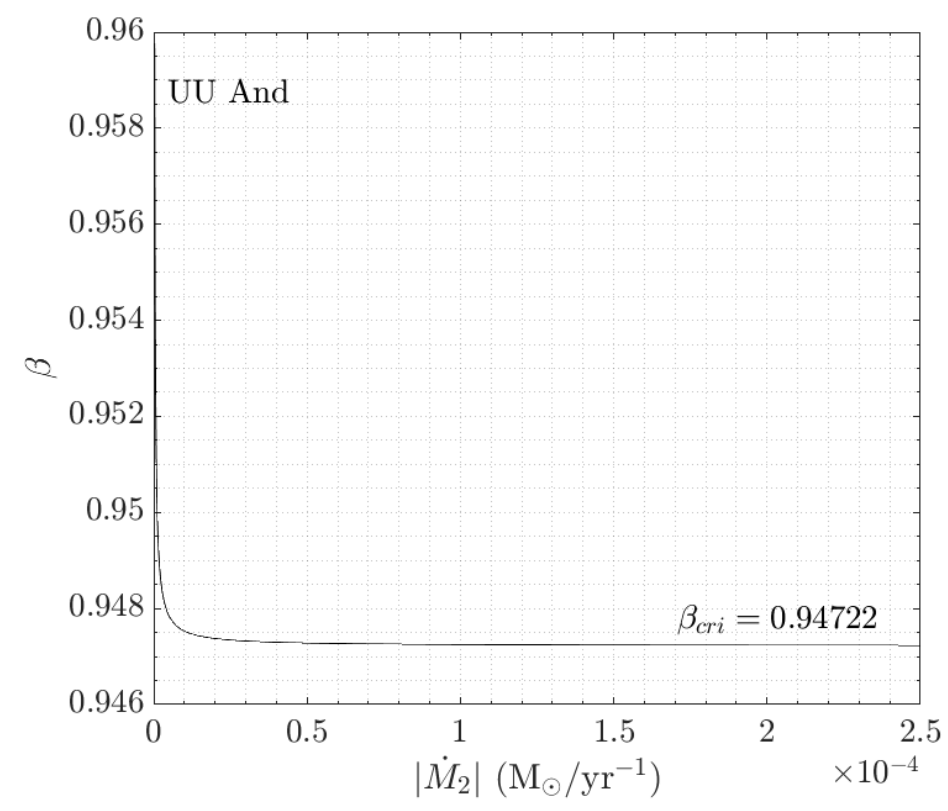

Figure 3. Change in fractional mass transfer rate $(\beta)$ with respect to $\dot{M}_{2}$.

By assuming non-conservative mass transfer and using Eqs. (3.1) and (3.2), the rate of transferred mass and that of mass loss from UU And was calculated in the interval of $10^{-4}-10^{-8}$ and $10^{-6}-10^{-14} \mathrm{M}_{\odot} / \mathrm{yr}$, respectively.

After subtracting parabolic variation from the O-C dataset, we detected clear sinusoidal variation in the O-C residuals (see lower panel of Figure 1). This cyclic variation was also investigated by Manzoori et al. (2015). They used minimum times observed from 1911 to 2008 and their O-C dataset was mostly visual and photographic. Since 27 new CCD and Pe minimum times have been published since 2008, we re-examined this cyclic variation with only CCD and Pe minimum times, which can be seen in the box in the lower panel of Figure 1. Our analysis is more precise than that of Manzoori et al. (2015) as we used more precise minimum times in order to model the cyclic O-C variation of UU And.

A sinusoidal O-C variation of UU And was also detected (see Section 2). The period, amplitude of cyclic variation and mass function were found to be 0.017( \pm 0.002$)$ days, $14.04( \pm 0.61) \mathrm{yr}$ and $0.173( \pm 0.037) \mathrm{M}_{\odot}$, respectively. Mass of the additional body was calculated to be $1.34( \pm 0.13) \mathrm{M}_{\odot}$ for $i_{12}=90^{0}$ and $1.62( \pm 0.15)$ $\mathrm{M}_{\odot}$ for $i_{12}=60^{0}$ using equation (2.4) (see Section 2). The light contribution of such a massive additional body show itself distinctly in the light curve of UU And. Unfortunately, no light contribution from a hypothetical third body was detected by Manzoori et al. (2015) when they analyzed the light curve of UU And. Due to this situation existing of a third body in the binary star system is not clear. Another possible mechanism which gives rise to cyclic $\mathrm{O}-\mathrm{C}$ variation may be magnetic activity in the system. In this case, the O-C diagram should show perfectly sinusoidal variation over time. On the contrary, our analysis show that the variation is not perfectly sinusoidal, i.e. $e=0.49$ (see Table 1). Therefore, in order to be certain about the existence of an additional body and/or possible magnetic cycle in the system, precise new minimum times and spectroscopic observations of the system are required. 


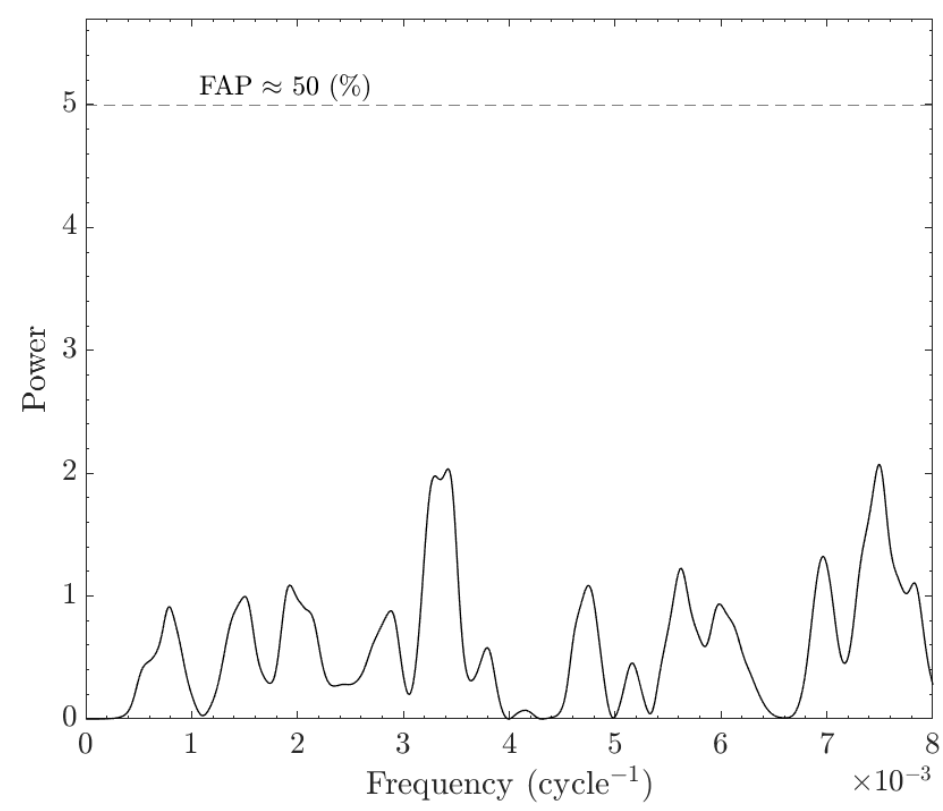

Figure 4. LS periodogram of O-C data after subtracting LTTE.

Lomb-Scargle (LS) periodogram was used to search for additional cyclic variations in the O-C data after subtracting LTTE (Lomb, 1976; Scargle, 1982). No signal above 50\% false alarm probability (FAP) level was detected in the LS periodogram.

\section{Conclusion}

This paper presents the recent O-C based orbital period analysis of UU And. Orbital period of UU And was found to be increasing with a rate of $\dot{P}=0.016$ (6) s/yr. Non-conservative mass transfer was assumed in order to explain the observed period increase. Sinusoidal variation was also detected in the O-C diagram of UU And. We concluded that this variation may be a sign of an additional body and/or possible magnetic cycle in the system.

\section{Acknowledgement}

The O-C database of the Czech Astronomical Society was used in research for this paper. We would like to thank Prof. A. Erdem for his comments. We would like to thank the anonymous reviewers for their valuable comments. We also would like to thank Mr. G.H. Lee for checking the English.

\section{Author Contributions}

Oğuz Öztürk: Conceived and designed the analysis.

\section{Conflicts of Interest}

We have no conflict of interest to declare.

\section{References}

Brancewicz, S. K., Dworak, T. Z. (1980). A catalogue of parameters for eclipsing binaries. Acta Astronomica, 30, 501-524. Retrieved from: http://articles.adsabs.harvard.edu/pdf/1980AcA....30.. 501B.

Erdem, A., Öztürk, O. (2014). Non-conservative mass transfers in Algols. Monthly Notices of the Royal 
Astronomical Society, 441(2), 1166-1176. https://doi.org/10.1093/mnras/stu630.

Irwin, J.B. (1959). Standard light-time curves. Astronomical Journal, 64, 149-155. https://doi.org/10. $1086 / 107913$.

Kreiner, J. M. (2004). Up-to-Date Linear Elements of Eclipsing Binaries. Acta Astronomica, 54(2), 207-210. Retrieved from: http://articles.adsabs.harvard.edu/pdf/2004AcA....54..207K.

Lomb, N. R., (1976). Least-Squares Frequency Analysis of Unequally Spaced Data. Astrophysics and Space Science, 39(2), 447-462. https://doi.org/10.1007/BF00648343.

Manzoori, D., Abbasvand, S., Najafinezhad, F. (2015). Analysis of the photoelectric light curve and the orbital period variations of the binary system UU Andromedae. Astronomische Nachrichten, 336(6), 570-576. https://onlinelibrary.wiley.com/doi/abs/10.1002/asna.201412196.

Pollacco, D. L., Skillen, I., Collier, C. A., Christian, D. J., Hellier, C., Irwin, J., Lister, T. A., Street, R. A., West, R. G., Anderson, D. R., Clarkson, W. I.., Deeg, H., Enoch, B., Evans, A., Fitzsimmons, A., Haswell, C. A., Hodgkin, S., Horne, K., Kane, S. R., Keenan, F. P., Maxted, P. F. L., Norton, A. J., Osborne, J., Parley, N. R., Ryans, R. S. I., Smalley, B., Wheatley, P. J., Wilson, D. M. (2006). The WASP Project and the SuperWASP Cameras. The Publications of the Astronomical Society of the Pacific, 118(848), 1407-1418. https://doi.org/10.1086/508556.

Qian, S. (2001). Possible Mass and Angular Momentum Loss in Algol-Type Binaries. IV. UU Andromedae and Z Persei. The Astronomical Journal, 122(3), 1561-1568. https://doi.org/10.1086/322179.

Scargle, J. D. (1982). Studies in astronomical time series analysis. II. Statistical aspects of spectral analysis of unevenly spaced data. Astrophysical Journal, 263, 835-853. https://doi.org/10.1086/160554.

Tout, C.A., Hall, D.S. (1991). Wind driven mass transfer in interacting binary systems. Monthly Notices of the Royal Astronomical Society, 253(1), 9-18. https://doi.org/10.1093/mnras/253.1.9.

Zasche, P., Liakos, A., Niarchos, P., Wolf, M., Manimanis, V., Gazeas, K. (2009). Period changes in six contact binaries: WZ And, V803 Aql, DF Hya, PY Lyr, FZ Ori, and AH Tau. New Astronomy, 14(2), 121-128. https://doi.org/10.1016/j.newast.2008.06.002. 